\title{
A100 INHIBITION OF MIR-182 DECREASES OVA-INDUCED ARTHRITIS IN MICE
}

Anna-Barbara Stittrich, Claudia Haftmann, ${ }^{1}$ Evridiki Sgouroudis, ${ }^{1}$ Zhuo Fang, ${ }^{2}$ Nikolaus Rajewsky, ${ }^{2}$ Hyun-Dong Chang, ${ }^{1}$ Andreas Radbruch, ${ }^{1}$ Mir-Farzin Mashreghi1 'Deutsches Rheuma-Forschungszentrum Berlin, a Leibniz Institute, Berlin, Germany; ${ }^{2}$ Max-Delbrück-Center for Molecular Medicine (MDC), Berlin, Germany; $A R$ and M-FM contributed equally to this work.

10.1136/ard.2010.148981.3

Background and objectives After antigenic stimulation, $\mathrm{T}$ helper lymphocytes have to expand clonally in order to generate a large pool of specific effector cells. This process is positively regulated by the interleukin-2 induced microRNA182 (miR-182), which inhibits the expression of the forkhead transcription factor Foxo1, a suppressor of $\mathrm{T}$ helper cell proliferation. Specific inhibition of miR-182 in T helper cells limits their expansion in vitro and in vivo. Therefore, the authors examined whether specific inhibition of miR-182 by antagomirs affects the ability of $\mathrm{T}$ helper cells to induce inflammation in a mouse model of OVA-induced arthritis.

Material and methods The authors treated OVA-specific T helper lymphocytes from DO11.10 mice with antagomir182 ex vivo and transferred them adoptively into Balb/c mice. One day after transfer the authors immunised with OVA. On day 14 after transfer, the authors injected OVA into the left knee joint and assessed knee swelling as a sign of inflammation. Knee joints were processed for histological examination.

Results Knee swelling was about $40 \%$ less after transfer of antagomir-182-treated T helper cells. Knockdown of miR-182 with antagomirs resulted in significantly lower histological scores for inflammation and tissue destruction. Infiltration of granulocytes and mononuclear cells into the inflamed tissue of the knee joint, as well as bone and cartilage destruction and the formation of pannus tissues, were lower in the mice given antagomir-182-treated cells than in the control group.

Conclusions The results showing less disease severity in a transfer model of OVA-induced arthritis open a new therapeutic avenue for the control of unwanted $T$ helper cell expansion in immune-mediated diseases by antagomirs. 